\title{
La percepción de investigadores brasileños sobre el depósito de la producción científica en repositorios institucionales de acceso abierto ${ }^{*}$
}

\section{Marilia Augusta de Freitas}

Doutora em Ciência da Informação, Universidade de Brasília. Bibliotecária en la Biblioteca Central de la Univeridad de Brasília, Brasília - Brasil.

marilia@bce.unb.br

orcid.org/0000-0003-0737-4542

\section{Fernando Cesar Lima Leite.}

Doutor em Ciência da Informação, Universidade de Brasília. Professor na Ciência da Informação.

Brasília-Brasil.

fernandodfc@gmail.com orcid.org/0000-0003-1998-2385

\begin{abstract}
Resumen
Este artículo tiene como objetivo presentar y discutir los resultados de la investigación sobre la percepción de los investigadores acerca del depósito de la producción científica en repositorios institucionales de acceso abierto. Los resultados presentados son parte de los resultados de una investigación mayor que tuvo como objetivo proponer las directrices para el depósito de la producción científica en repositorios institucionales de acceso abierto con base en la percepción de los distintos actores que participan del sistema de comunicación científica. Desde el punto de vista metodológico el estudio tiene un propósito descriptivo y un abordaje metodológico cualitativo, dado que la propuesta de las directrices tuvo por base las percepciones de los actores involucrados. Como método de investigación fue adoptado el levantamiento de información, el cual, por su parte, fue operacionalizado con la adopción de la técnica de la entrevista semiestructurada. Entre las conclusiones destacan que las principales motivaciones para depositar la producción científica en repositorios institucionales son diversificadas en función de las áreas del conocimiento. Entre ellas están la conciencia de los beneficios del acceso abierto, la obligatoriedad del depósito, los estímulos institucionales y la vinculación a las disciplinas culturalmente propensas al depósito.
\end{abstract}

Palabras clave: sistema de comunicación científica, repositorios institucionales, acceso abierto.

Cómo citar este artículo: Freitas, M. A., \& Leite, F. C. L. (2019). La percepción de investigadores brasileños sobre el depósito de la producción científica en repositorios institucionales de acceso abierto. Revista Interamericana de Bibliotecología, 42(2), 159-173. doi: 10.17533/udea.rib.v42n2a04

Recibido: 2018-03-19 / Aceptado: 2019-04-09

* El presente texto es un avance del proyecto "Acesso aberto à informação científica na América Latina e Caribe: insumos para a gestão e comunicação da informação científica”, de la línea del Grupo de Investigación Publicações Eletrônicas do Programa de Pós-Graduação em Ciência da Informação da Faculdade de Ciência da Informação da Universidade de Brasília, Distrito Federal, Brasil. 


\section{The Perception of Brazil- ians Researchers on the Deposit of Scientific Production in Open Access Institutional Repositories}

\begin{abstract}
This work aims to present and discuss results of research about the perception of researchers regarding the deposit of scientific production in open access institutional repositories. The results presented compose results of a larger research whose purpose was to propose guidelines for the deposit of scientific production in open access institutional repositories based on the perception of the different actors that participate in the scholarly communication system. From the methodological point of view, the study has a descriptive purpose and a qualitative methodological approach, since the proposal of the guidelines was based on the perceptions of the actors involved. As a research method, the survey was adopted, which was operationalized with the adoption of the semistructured interview technique. Among the conclusions, the main motivations for depositing scientific production in institutional repositories are diversified according to the areas of knowledge. Among them are the awareness of the benefits of open access, compulsory deposit, institutional incentives and the link to culturally deposit-prone disciplines.
\end{abstract}

Keywords: Scholarly communication system, institutional repositories, open access.

\section{Introducción}

Los repositorios institucionales han sido utilizados como estrategia de acceso abierto a la información científica con la finalidad de promover la amplia circulación y visibilidad de los resultados de la investigación. El éxito de tal estrategia depende concretamente de la acción del depósito de la producción científica en repositorios institucionales. Tal proceso constituye un espectro diverso de posibilidades que convergen para una única finalidad, el poblamiento de los repositorios institucionales de acceso abierto a la información científica. Como un nuevo proceso del complejo sistema de comunicación científica, el depósito de la producción científica en repositorios institucionales depende principalmente de los investigadores. En ese contexto, los investigadores son actores centrales del proceso de depósito, debido a que son productores y responsables intelectuales por los resultados de la investigación. El estudio de la percepción que tales actores poseen del depósito de la producción científica y de ese proceso es fundamental para la comprensión y la propuesta de las directrices que contribuyan a potenciar el poblamiento de los repositorios institucionales.

Este artículo tiene como objetivo presentar y discutir los resultados de la investigación sobre la percepción de investigadores brasileños acerca del depósito de la producción científica en los repositorios institucionales. Los resultados presentados son parte de los resultados de una investigación mayor que tuvo como objetivo proponer las directrices para el depósito de la producción científica en repositorios institucionales de acceso abierto basado en la percepción de los distintos actores que participan del sistema de comunicación científica (Freitas, 2015).

\section{Breve fundamentación teórica}

Independientemente del método utilizado para depositar la producción científica en un repositorio de acceso abierto - autoarchivo o depósito mediado-, la decisión de hacerlo corresponde al investigador. Esa toma de decisiones sufre, sin embargo, la influencia de las diferentes tendencias, procedentes sobre todo de los otros actores del sistema de comunicación científica. Además de eso, otras cuestiones relacionadas con las diferencias disciplinares influencian en la decisión de hacer, o no, el depósito. Foster y Gibbons (2005) recuerdan, al respecto, que ciertos docentes-investigadores de universidades se incomodan al ser obligados a ejecutar tareas que van más allá de la investigación, como las lecturas, la redacción de artículos, los proyectos e informes, la participación en foros de discusión, entre otras. O sea, al ser considerada una actividad ajena a las actividades típicas de la investigación, el depósito de la producción científica puede, tal vez, quedar descuidado.

En el estudio que involucró a profesores de 17 universidades de los Estados Unidos que poseían repositorios institucionales, Kim (2008) analizó el impacto de las características individuales del investigador sobre las motivaciones que tenía para realizar el autoarchivo. Según el autor, el número de publicaciones, la posición profesional 
y el cargo que un investigador ocupa en la institución son factores que influencian definitivamente en la toma de decisiones. Entre los resultados de su estudio, Kim llegó a proponer un modelo que presenta las principales variables que afectan a las decisiones de los investigadores en lo que concierne al autoarchivo (Figura 1).

En el modelo de Kim (2008), los factores relacionados al costo influencian negativamente la decisión de autoarchivo, mientras que los relacionados a los beneficios generados influencian positivamente esa práctica. El modelo supone que la confianza y la cultura del autoarchivo como factores contextuales, en conjunto con el número de publicaciones como una particularidad individual, están positivamente relacionadas con la decisión por el autoarchivo.

Kim (2008) basó su trabajo en un modelo producido por Kankanhalli, Tan y Kwok (2005), que tenía por objetivo explicar el uso de los repositorios electrónicos por los "colaboradores" del conocimiento, los cuales incorporan concepciones de la teoría del cambio social y de la teoría del capital social. A ese modelo, Kim (2008) agregó las particularidades individuales de cada investigador que podían afectar determinantemente su motivación para el autoarchivo. El autor concluyó que los profesores raramente optan por las innovaciones (modificaciones en las prácticas de la comunicación científica) a la hora de publicar sus investigaciones, dado que el sistema de comunicación científica en vigor está profundamente enraizado en los valores académicos.

De esta forma, más que enriquecer el registro de metadados, la integración de sistemas actúa como factor de estímulo para el depósito, en la medida en que facilita tanto el autoarchivo propiamente dicho como el depósito mediado. En ese sentido, la integración de sistemas es un esfuerzo necesario para facilitar el depósito y reducir el tiempo necesario empleado en el registro de cada uno de los ítems por los investigadores y en la descripción de los ítems depositados por parte del gestor del repositorio. Aun en concordancia con Martins, Nolasco y Silva (2013), en el contexto universitario, es esencial una gestión integrada de la información científica en lo que concierne a la evaluación, al acceso, a la visibilidad, a la preservación, a la integración y a la interoperabilidad.

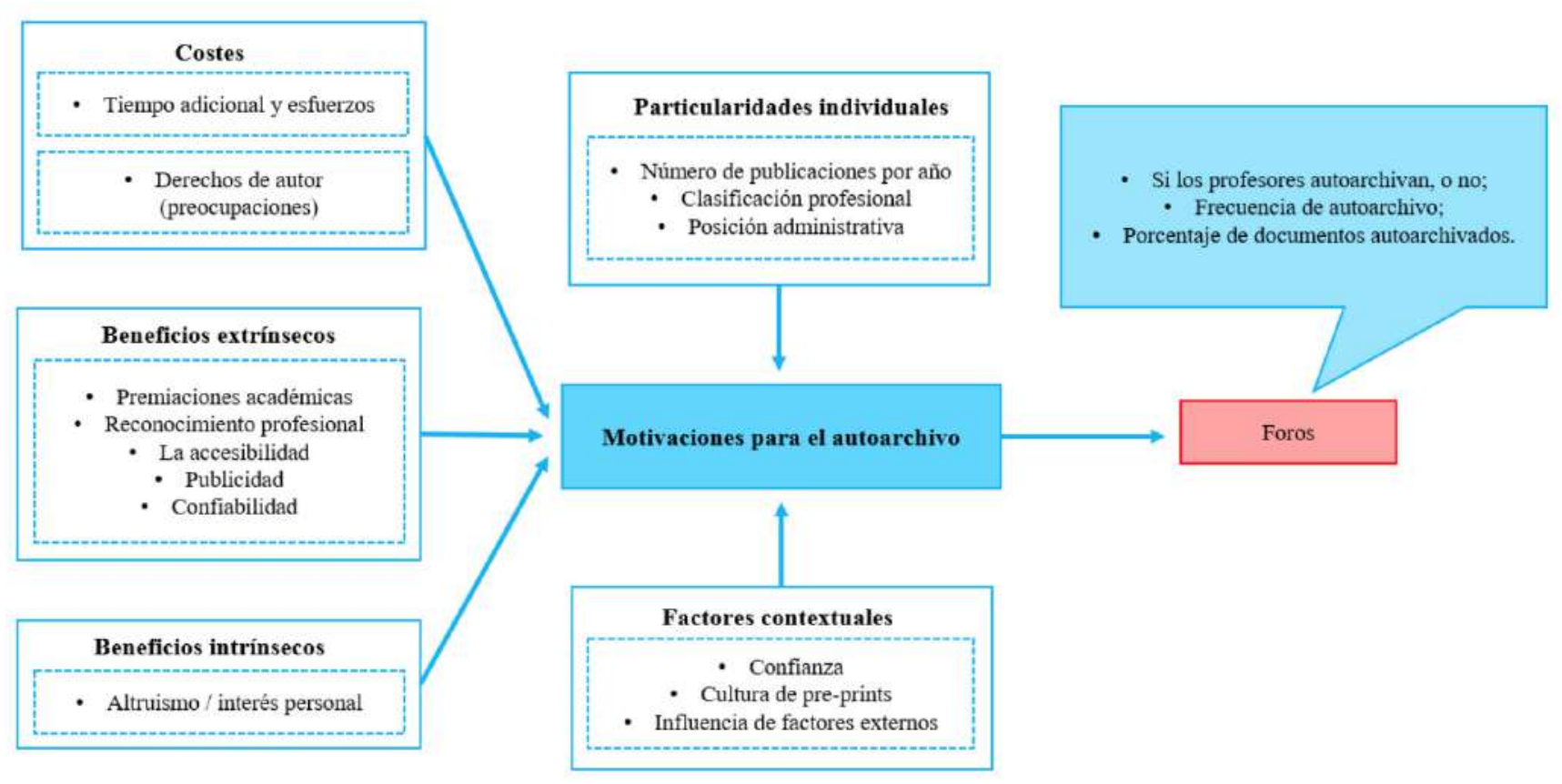

Figura 1. Modelo de los factores que afectan el comportamiento para el autoarchivo.

Fuente: Kim (2008, p. 23, traducción nuestra). 
Otra dimensión crucial relacionada con el depósito de la producción científica es la referente a los derechos de autor. La cuestión legal ejerce una innegable influencia sobre la toma de decisiones con relación al depósito de la producción científica en repositorios institucionales. Con el propósito de colaborar con la viabilidad del autoarchivo sin infringir en los derechos de autor de las publicaciones, surgieron directorios, como el Sherpa/ Romeo, de la Universidad de Nottingham, en Reino Unido, y Diadorim, una iniciativa brasileña, coordinada por el Instituto Brasileño de Información en Ciencia y Tecnología (IBICT). Ambos directorios tienen como objetivo suministrar información relacionada con las políticas de almacenamiento de los artículos en repositorios institucionales. La cuestión de los derechos de autor aún genera muchas dudas entre los investigadores, en función de la complejidad de los aspectos involucrados.

Antelman (2006) mostró preocupación con la relación entre la práctica del autoarchivo y el respeto a los derechos de autor. Según la autora, la mayoría de los contratos de derechos de autor no mencionan o son ambiguos en lo referido a los derechos de depósito en repositorios institucionales y a la versión que puede ser depositada. Aun cuando se esté de acuerdo con el depósito, son utilizados términos imprecisos para describir las versiones (por ejemplo, "el trabajo", "el paper", "la contribución"), que no facilitan al autor el decidir en qué versión deberá autoarchivar. Además de eso, la mayoría de los editores exige una cesión de derechos patrimoniales en diferentes puntos del proceso de evaluación, dependiendo de la revista científica. Además, muchos autores ni siquiera tienen idea de los términos de sus contratos de derechos de autor; y otros creen que son ellos los únicos detentores de los derechos patrimoniales de sus propios trabajos. A eso se suma el hecho de que, aún entre los autores que muestran un buen conocimiento sobre los derechos de autor, o son conscientes de las exigencias de los editores o son muchos los que los descuidan.

\section{Metodología}

Los resultados de la investigación realizada y relatada en este artículo constituyen parte de una investigación más amplia que tuvo como objetivo proponer directrices para el depósito de la producción científica en los repositorios institucionales de acceso abierto con base en la percepción de los distintos actores que participan del sistema de comunicación científica. Este artículo relata exclusivamente la percepción de los investigadores sobre el depósito de la producción científica en repositorios institucionales.

Tal como es descrito por Freitas (2015), la investigación tuvo un propósito descriptivo y un abordaje metodológico cualitativo, toda vez que la propuesta de las directrices se basó en las percepciones de los actores involucrados. Como método de investigación se adoptó el levantamiento (Babbie, 1999). Fowler (2011) considera que la principal forma de recolectar datos en estudios que se valen del método de levantamiento es hacer preguntas a los entrevistados. Por esta razón, la técnica adoptada para la recolección de datos fue la entrevista semiestructurada. Para hacer operativo el proceso de obtención de datos, fue elaborado y aplicado un guion de entrevista, constituido de 11 preguntas abiertas. Las entrevistas fueron realizadas en el periodo de mayo de 2015 a octubre de 2015 .

La definición de la muestra de la investigación como un todo fue hecha de manera estratificada, o sea, para cada actor del proceso de comunicación científica fueron definidos criterios específicos a partir de los cuales los sujetos de la investigación fueron seleccionados. Se optó, entonces, por el muestreo intencional no probabilístico. Considerando que este artículo trata solo de los resultados del estudio referente a las percepciones de los investigadores, fueron adoptados los siguientes criterios para las definiciones de los entrevistados: representar divisiones del conocimiento y disciplinas; tener currículo Lattes actualizado el último año; ser doctor; estar vinculado al programa de posgrado; poseer producción significativa para los estándares del área (en consonancia con los Documentos de Áreas de la Capes). Fueron realizadas ocho entrevistas con investigadores de diferentes áreas del conocimiento y disciplinas, a considerar: ciencias (física, ciencias de la salud y biología), ciencias sociales y humanas (sociología, antropología y administración) y artes y humanidades (historia y geografía), todos vinculados a la Universidad de Brasilia.

De las entrevistas resultaron archivos de audio que fueron transcritos y generaron textos por grupos de 
actores. Los textos fueron resumidos y categorizados manualmente y, luego, se identificaron los factores que inhiben y estimulan el depósito de la producción científica de acuerdo con cada grupo estudiado. En este artículo se presentan los resultados relacionados con el grupo de investigadores.

\section{Análisis y discusión de los resultados}

El último grupo de actores entrevistados fue el de los investigadores. Son esos actores los que inician el flujo de la comunicación científica y son responsables por la producción del conocimiento. La decisión de depositar su producción científica, por parte de los investigadores, es influenciada positiva o negativamente por los demás actores del proceso de comunicación científica. La visión de los investigadores con relación a la temática es analizada a continuación, así como sus opiniones con relación al modo y el rol desempeñado por los demás actores que pueden interferir directamente en la decisión de depositar la producción científica en repositorios institucionales de acceso abierto.

\subsection{Percepciones respecto al acceso abierto}

Los investigadores entrevistados demostraron conocer acerca de toda la problemática que envuelve el movimiento del acceso abierto, tanto como se mostraron favorables a él. Algunos investigadores entrevistados afirmaron que es un camino sin retorno y algo que se ha vuelto cada vez más natural según lo transcrito a continuación.

Veo el acceso abierto como una decisión sin retorno. Digo sin retorno en el buen sentido de la palabra, en la medida en que el acceso abierto a la comunicación científica, especialmente para países en desarrollo, es una alternativa, es el camino para la expansión del conocimiento científico y, por lo tanto, es el camino para ampliar el propio proceso de desarrollo científico del país. En líneas generales, lo veo de esa forma, o sea, yo soy un defensor del acceso abierto. (Investigador 3)

Creo que actualmente es el camino natural para exponer los resultados de tu investigación [...] Entonces, creo que el futuro de la información científica está en permitir el libre acceso a las personas. (Investigador 7)
Otros investigadores hicieron énfasis en la cuestión de la democratización de la ciencia, sin embargo, resaltaron que no es algo fácil de establecer, pues existen discusiones sociológicas y políticas en torno a toda la problemática que envuelve el hacer disponible los resultados de la investigación en acceso abierto.

El [acceso abierto] es un instrumento de democratización, permite popularizar el conocimiento de la ciencia, asume que la educación y la investigación son bienes esenciales, pero trae una serie de desafíos sobre las concepciones del conocimiento como la propiedad del conocimiento dentro de los repositorios privados. (Investigador 5)

Defiendo el acceso abierto. Lo defiendo radicalmente, o sea, desde mi punto de vista, toda la producción intelectual en el mundo debería ser abierta. (Investigador 8)

Las opiniones presentadas por los entrevistados siguen los principios delineados por la necesidad de remover las barreras del modelo tradicional de comunicación científica, así como deshacer las barreras que impiden el acceso a la literatura científica, con el fin de acelerar la investigación, fortalecer la educación y difundir el conocimiento de manera general (BOAI, 2002).

\subsection{Producción depositada en repositorios institu- cionales de acceso abierto y motivación para el depó- sito}

Se consultó a los investigadores si ya tenían su producción depositada en algún repositorio digital y por qué motivo decidieron hacerlo. Todos los entrevistados, con la excepción del Investigador 7, tenían algún documento de su autoría en algún tipo de repositorio. Los motivos por los cuales esos investigadores decidieron depositar su producción en un repositorio de acceso abierto son los más diversos, conforme muestran a continuación las transcripciones de las entrevistas:

Tengo parte de mi producción depositada en el repositorio institucional de la UnB; decidí depositar, pues es un medio de corroborar lo que publiqué y es una forma de divulgar lo que uno está haciendo. Porque si yo tuviera alguna información y solo es utilizada en la UnB para mí no ayuda mucho. (Investigador 1) 
Mis trabajos de máster y doctorado están en el repositorio de la Unesp. Está allí porque es obligatorio. (Investigador 2)

Tengo textos que están en el repositorio de la Universidad de Brasilia, en verdad no me preocupo mucho en buscar el repositorio para depositar algún texto, porque la mayor parte, si no el $100 \%$, de mis textos son artículos publicados en revistas científicas. (Investigador 3)

Tengo publicaciones que están en revistas científicas on-line de acceso abierto. Algunas están en el repositorio de la UnB. (Investigador 4)

Ese caso, ya lo tuve en la UnB, y en esa ocasión lo decidí no solo por la vinculación institucional a la Universidad de Brasilia. [...] Pero especialmente debido al bibliotecario. No hubiera tenido la iniciativa individual si no tuviera un agente motivándome. Me parece también que es muy importante la figura del administrador del repositorio, sea el bibliotecario o quien fuere, el científico de la información, no sé quién es la figura especializada, motivando al científico, al intelectual a decir: "eso tiene sentido, aquí está el papel". (Investigador 5)

Allá ellos tenían un repositorio de tesis y disertaciones. Donde yo defendí la maestría, que fue en Río de Janeiro, mi disertación está disponible, y donde yo defendí el doctorado, en Río Grande del Sur, mi tesis también está disponible. (Investigador 6)

A mí, específicamente, nunca me lo reclamaron y nunca pedí que mis trabajos aparezcan, precisamente porque uno anda muy enfocado, tal vez, en la producción científica, en las revistas científicas. No lo sé, tal vez, ni sé los caminos para llegar a esos repositorios. Por eso, creo que, si uno es motivado, tendría el mayor gusto en divulgar mi información en ese tipo de medio, digamos, que hasta existen algunos aspectos interesantes con relación a eso. (Investigador 7)

Tengo bastante. Casi toda. En realidad, es una práctica del área. Es una práctica usual de nuestra área de física, antes de hacer la publicación. Existen algunos repositorios en el mundo que son abiertos y existen acuerdos con la mayoría de las revistas, la mayoría quiere decir todas, no conozco una que se niegue, o sea, que exija que no sea depositado en el repositorio, de hecho, las más influyentes exigen que pase por el repositorio primero, hasta porque es un componente, una especie de filtro, de referente para la comunidad. (Investigador 8)

Como es posible notar en la conversación con los entrevistados, la decisión de hacer disponible su producción en algún tipo de repositorio pasó por motivaciones diferentes. Dos investigadores (investigadores 2 y 6) citaron que sus trabajos de maestría y doctorado están en el repositorio institucional de la universidad en la que fueron defendidos, por causa de la obligatoriedad.

Los investigadores 1, 3, 4 y 5 tienen parte de su producción disponible en el repositorio institucional de la universidad en la que trabajan. La decisión de esos investigadores de hacer disponible su producción fue motivada por el conocimiento del investigador sobre la importancia de que su producción esté en acceso abierto (Investigador 1), así como por el papel del bibliotecario como agente motivador (Investigador 5). Los investigadores 3 y 4 , a pesar de tener documentos disponibles en el repositorio de su institución, no fueron motivados por la misma forma. Probablemente aquellos documentos que son de acceso abierto estén disponibles por iniciativa del bibliotecario. El Investigador 7 afirmó nunca haber sido motivado a depositar su producción en un repositorio institucional, y su principal preocupación es la publicación en revistas científicas, pero tendría el agrado de hacer disponible su producción en el repositorio institucional si fuera invitado a hacerlo.

El Investigador 8, por pertenecer a un área que tiene como cultura el depósito en repositorios temáticos, posee casi toda su producción disponible en repositorios. Esa cultura se dio por el hecho de que las áreas de ciencias exactas necesitaron enfrentar los problemas que existían con el sistema tradicional de comunicación científica. Esas áreas necesitaban que los resultados de la investigación estuvieran disponibles lo más ampliamente posible, por lo tanto, desarrollaron soluciones como los repositorios temáticos (Costa, 2005).

\subsection{Los factores que estimulan e inhiben el depósito de la producción científica en los repositorios insti- tucionales de acceso abierto}

Así como fue descrito para los demás grupos de actores analizados, son muchos los factores que pueden estimular o inhibir el depósito en repositorios instituciona- 
les de acceso abierto. La principal motivación abordada por los investigadores es la divulgación del repositorio y de los beneficios asociados al depósito, según los comentarios siguientes.

Cada inicio de semestre, principalmente para los coordinadores de especialización y para los programas de posgrado, se tendría una mini presentación del bibliotecario... (Investigador 1 )

Considero que una mayor divulgación. Hacer un trabajo de divulgación... (Investigador 2)

El factor de estímulo, diría que es la visibilidad del investigador, por ejemplo, si coloco mis textos en el repositorio de la Universidad de Brasilia, me haría más visible en la comunidad, mis textos tendrían más accesos. (Investigador 3)

Mira, creo que, probablemente, otros investigadores se pueden interesar en hacerlo y, probablemente, también creo que eso estimularía a los propios alumnos a depositar sus trabajos, quiero decir, eso podría ser, de hecho, ya lo es, una especie de red. (Investigador 4)

Creo que necesitamos, de una forma mucho más amplia, como investigadores, estudiantes, en la graduación y posgrado, información sobre el papel del repositorio en las bibliotecas virtuales, de cuál es el sentido de una revista científica electrónica, de la comunicación de acceso libre e irrestricto, de cuál es la importancia de eso. (Investigador 5)

La presión de los grandes autores, de los más citados, por ejemplo, al divulgar su trabajo abiertamente.(Investigador 6 )

Creo que cabría justamente una acción en ese punto, por eso creo que el principal punto es que la biblioteca cree, junto al programa de posgrado, tal vez un día, un simposio, un workshop, puede ser para todos los programas, digamos, que vamos a reunir a todos los coordinadores y a quienes estén interesados, entonces usted monta un workshop (repositorio y su integración con la sociedad, y su inserción en la sociedad, divulgación social de la información), para motivar los programas de posgrado para hacerlo y a la vez para mostrar que la institución ya tiene una vía para eso. Por ello, creo que ese sería el principal punto, la divul- gación y la motivación de los programas de posgrado de insertarse en esa área, porque tengo la certeza de que ningún investigador está en contra la divulgación de sus trabajos a la comunidad. (Investigador 7)

Pienso que la manera de hacer el upload del artículo sería un factor de estímulo. Acercar los repositorios temáticos. (Investigador 8)

Se desprende del análisis de las entrevistas que los factores relacionados con los beneficios de los repositorios institucionales son las principales influencias en la práctica del depósito de la producción científica. Ese resultado concuerda con los resultados obtenidos por Kim (2008), que concluyó que los beneficios extrínsecos, como la accesibilidad, la publicidad y la fiabilidad, son factores determinantes en la toma de decisiones del investigador para depositar su producción científica en el repositorio de la institución. Sin embargo, se entiende, por las respuestas obtenidas, que los investigadores solamente conocieron los beneficios y las ventajas de hacer disponible su producción en el repositorio institucional si hubo una divulgación de ese servicio.

Cuando preguntamos sobre cuáles factores podrían inhibir el depósito de la producción científica en los repositorios institucionales, las respuestas no fueron homogéneas. Algunos investigadores citaron la falta de conocimiento como el principal factor, mientras otros creen son todas las cuestiones vinculadas a la propiedad intelectual y los derechos de autor. Y uno de los investigadores afirmó que era la estructura (el formato) requerida por el repositorio, el modo como los depósitos son hechos, pues su mayor preocupación es que sea algo simple y dinámico. Los siguientes testimonios lo confirman:

Creo que es la falta de conocimiento, de divulgación. (Investigador 1)

Creo que no hay ningún impedimento, solo el aspecto de la falta misma de divulgación. (Investigador 2)

Si algún conocimiento que fue producido depende de alguna etapa previa antes de ser divulgado, en el sentido de proteger algún derecho: derechos de propiedad, derechos intelectuales, derecho de marca o algo parecido. (Investigador 3) 
Lo que me inhibiría es lo que me inhibe en todo, en cualquier lugar del mundo, la burocracia. (Investigador 4)

Primero, la concepción de la propiedad intelectual, de la idea de que la producción es una propiedad y no es solo del investigador. [...] no lo traduciría por mí, pero como una sociología del fenómeno en el Brasil, una mala concepción de que estar en el repositorio sería un desmérito. No desmérito, la palabra no es esa, pero la dificultad de comprensión de que las redes virtuales son solo un medio, pero que el repositorio, él es un espacio propio de divulgación de la información, o sea, la no comprensión de lo que es uno repositorio... (Investigador 5)

Me parece que los grandes portales de ciencia del mundo, [...] creo que ellos actúan contra eso. Porque, a fin de cuentas, esos portales muchas veces pagan a los evaluadores, ellos pagan para mantener la web con una excelencia de interfaz, tienen una serie de cuestiones técnicas vinculadas a esos portales que exigen recursos regulares. (Investigador 6)

Pienso que principalmente es por la falta de conocimiento que existe. Por eso, muestro mi falta de conocimiento en ese punto realmente, pero uno tampoco fue incentivado a hacerlo, porque a uno siempre se le exigió la producción científica, la producción científica, la producción científica. (Investigador 7)

Entonces, una cosa que inhibe es la estructura de él. Él tiene que tener una estructura, diría, vamos a decir así genéricamente dinámica, muy dinámica, muy práctica cómo lo ArXiv. (Investigador 8)

Es posible percibir, por las respuestas obtenidas, que los investigadores están preocupados con la publicación en revistas científicas, porque ellos necesitan producir como parte de sus atribuciones como docentes de una universidad, sin embargo, no existe ninguna preocupación en divulgar esa producción en repositorios institucionales. Eso puede ser explicado por el desconocimiento acerca del servicio debido a la falta de divulgación, que fue citada como uno de los factores que inhibe el depósito. Algunos aspectos abordados por los investigadores entrevistados están en concordancia con las conclusiones obtenidas por Kim (2008) en su estudio. El factor tiempo y las cuestiones burocráticas que conllevan el depósito, así como cuestiones relacio- nadas con los derechos de autor son centrales para la toma de decisiones con relación al depósito de la producción científica en repositorios institucionales.

Otro aspecto abordado por uno de los investigadores entrevistados (Investigador 8) es lo relacionado con el modo como los repositorios institucionales están estructurados. Por tratarse de un investigador perteneciente a la disciplina que posee una cultura de depositar en repositorios temáticos, su concepción es que los repositorios institucionales necesitan ser más fáciles, a ejemplo de ArXiv (repositorio temático en el área de física, matemática, ciencia de la computación, entre otras), en el cual el área de física tiene por tradición depositar su producción.

En ese sentido, al identificar las diferentes prácticas para el depósito de la producción científica, los gestores de repositorios pueden crear nuevas estrategias para la operación de sus repositorios y para el desarrollo de políticas de archivado (Xia \& Sun, 2007). El abordaje de esas políticas necesita considerar las diferencias disciplinares, las colaboraciones, las prioridades de los departamentos, el apoyo disponible, la edad, el sexo, el nivel en la carrera y el conocimiento tecnológico, factores que influencian en la práctica del depósito (Covey, 2008). La evaluación de ese conjunto de factores, por parte de los gestores de los repositorios, podrá contribuir a la divulgación del repositorio ante la comunidad académica, así como para la oferta de servicios que promuevan el depósito de la producción científica basados en las particularidades individuales de cada investigador o en las peculiaridades de cada área. La Figura 2 representa, a partir de las respuestas obtenidas en las entrevistas con los investigadores, los aspectos categorizados que estimulan e inhiben el depósito.

\subsection{Política de depósito obligatorio en repositorios institucionales de acceso abierto}

Los investigadores entrevistados fueron consultados sobre cómo reaccionarían si fuera adoptada una política de depósito obligatorio para los repositorios institucionales de la universidad en la que trabajan. Tres investigadores entrevistados afirmaron que eso debería hacerse, y uno de ellos afirmó que reaccionaría de manera natural, o sea, no tendría problema en hacer el depósito de su producción en el repositorio institucional. 


\section{Factores que motivan}

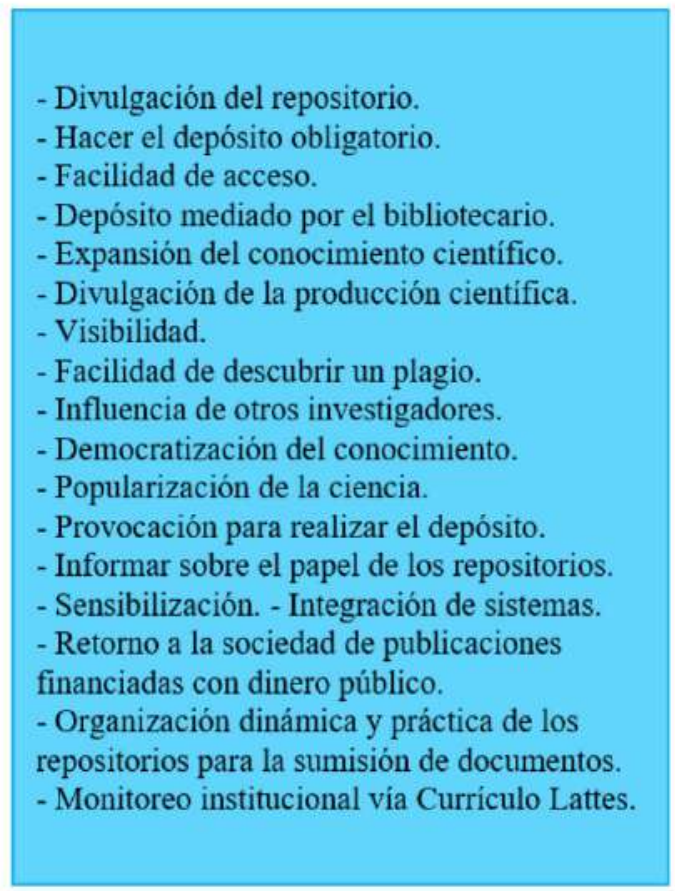

Factores que inhiben

- Falta de divulgación de los repositorios.

- Depósito obligatorio.

- Cuestiones relativas a los derechos de autor.

- Interrogaciones relativas al plagio.

- Burocracia.

- El investigador necesita completar muchos sistemas.

- Propiedad intelectual.

- Concepción de que aquello que está disponible en repositorios no posee calidad. - Desinformación sobre el papel de los repositorios.

- Grandes portales de ciencia del mundo, que visan logro con publicaciones científicas.

- Falta de conocimiento de la existencia de los repositorios.

- Falta de divulgación de los repositorios.

- Manera de hacer upload de los archivos en el repositorio.

Figura 2. Representación gráfica de los factores que motivan y los factores que inhiben el depósito en repositorios institucionales desde el punto de vista de los investigadores.

Fuente: elaboración propia.

Considero que, mientras no se haga eso, el personal no va a depositar. Tiene que hacerse. (Investigador 1)

Reaccionaría de forma natural, muy tranquilo. Haría eso sin ningún problema. (Investigador 2)

Considero que si usted está dentro de la universidad, usted hace investigación usando la infraestructura de la universidad [...] de cierta forma, usted está usando el nombre de la universidad en todo lo que fue construido para facilitarte eso, entonces creo que eso es una cláusula que ya debería ser obligatoria, ya implícita de usted divulgar sus resultados y la universidad puede usar tus resultados. (Investigador 7)

Los demás investigadores afirmaron que reaccionarían mal a una política de depósito obligatorio, pues creen que esa no sería la mejor forma de viabilizar el depósito en el ámbito de la institución.

En cuanto a que sea impuesto, creo que no va a funcionar. Creo que tienen que dejar al investigador libre para que escoja donde él quiere divulgar sus trabajos. (Investigador 2)
Yo reaccionaría muy mal, porque creo que en una democracia no se debe obligar a las personas a hacer nada. (Investigador 4)

Mi ejemplo no es bueno, porque yo ya me sometí a eso. Pero imaginando que yo no tuviera, imaginando que yo supiera menos, yo tal vez tuviera una resistencia por el carácter obligatorio. Yo tendría que ser sensibilizada sobre lo que es lo repositorio, como él existe en otros países, cual el significado de eso. (Investigador 5)

La expresión "obligatorio" es muy pesada, principalmente tratándose de universidad pública. (Investigador 6)

Obligatorio no lo encuentro razonable [...] Creo que tienen que crear una cultura del depósito, no como una obligación. (Investigador 8)

Las respuestas obtenidas concuerdan con lo que afirma Xia et al. (2012), en que, a pesar del éxito obtenido con las políticas de depósito obligatorio, tal efecto del mandato es imprevisible. Esa imprevisibilidad puede darse, sobre todo, por los investigadores que tengan re- 
sistencia a la palabra "obligatorio", según muestran las transcripciones anteriores. De acuerdo con los investigadores entrevistados, si existiera la adopción de una política obligatoria de depósito, la reacción de la comunidad de investigadores tiende a ser negativa. Aunque pueda haber mayor éxito en los depósitos cuando hay una política que obliga, se percibe por el análisis de los datos obtenidos que es importante, antes de la institucionalización de una política de depósito obligatorio, sensibilizar a la comunidad académica acerca del papel de los repositorios institucionales, de las ventajas y los beneficios de hacer disponible la producción científica en esos repositorios.

Esta sensibilización es necesaria para que se conozcan las demandas de la comunidad académica, considerando que las necesidades de las universidades no son las mismas, además de que existen diferencias en el comportamiento de los investigadores de diferentes disciplinas. Las políticas necesitan ser elaboradas de modo que reflejen esas necesidades. Además de eso, los gestores de los repositorios institucionales necesitan desarrollar estrategias para hacer aplicables esas políticas. La investigación elaborada por Xia et al. (2012) muestra que la proposición "una vez creado, ellos van a depositar" no es realista.

Siendo así, se entiende, por lo que expresaron los investigadores, que el camino más viable para hacer efectivo el depósito en repositorios institucionales es la divulgación del servicio. Se debe crear una cultura de depósito para que los investigadores la tengan como un hábito, en paralelo al trabajo de divulgación, crear los mecanismos de discusión para la adopción de políticas obligatorias, las cuales deben ser implementadas en el ámbito de la institución.

\subsection{Autoarchivo o depósito mediado}

En el caso de que los investigadores tomaran la decisión de depositar su producción científica en un repositorio institucional, se preguntó si ellos mismos harían el depósito o delegarían la tarea a terceros. La mayoría de los entrevistados afirmó que delegarían. Muchos afirmaron además que el bibliotecario posee la capacidad para realizar tal tarea, y otros creen que debería ser algo automatizado, con monitoreo a través del currículo Lattes.
Solamente un investigador afirmó que, a pesar de preferir delegar, él haría el autoarchivo si el sistema de depósito fuera más simplificado. Las transcripciones a continuación traen las respuestas de los entrevistados con relación a esa pregunta.

Encuentro mejor autorizar al bibliotecario. Porque así facilita para uno. Todas las veces que pudiera el bibliotecario ya sabe que está autorizado. (Investigador l)

Delegaría al bibliotecario, porque creo que es mucho trabajo. (Investigador 2)

Mira, yo entiendo que eso ahí podría ser hecho de forma instruccional. ¿A qué yo llamo de forma instruccional? La propia UnB podría tener un equipo que identificaría las publicaciones de sus profesores y servidores de modo general... (Investigador 3)

[...] en mi caso, que ya tengo muchos años de UnB, creo que solicitaría la ayuda del bibliotecario para hacerlo conmigo, si fuera posible un bibliotecario o alguien con conocimiento semejante. (Investigador 4)

Yo prefiero autorizar. Pero lo más razonable para mí sería un monitoreo institucional. Una vez que yo actualizo mi Lattes, el Lattes informaría al repositorio... (Investigador 5)

Prefería autorizar, porque realmente el tiempo es muy escaso. (Investigador 6)

Bien, yo voy a ser sincero con usted, yo prefería que hubiera alguien responsable para eso. (Investigador 7)

Si hubiera alguien para hacer eso para mí sería óptimo. (Investigador 8)

Las respuestas muestran que, entre los investigadores entrevistados, difícilmente la cultura del autoarchivo se establecería. Por diversos motivos, los investigadores no quieren, o no pueden hacer el autoarchivo y prefieren delegar esa tarea. El depósito mediado es la preferencia de los investigadores, a pesar de que los repositorios sean construidos para el autoarchivo (Cooke, 2007).

En ese sentido, como el depósito mediado es el más usual y los bibliotecarios son los más calificados para 
realizar la tarea, es importante que haya inversiones en la biblioteca para garantizar que los bibliotecarios tengan condiciones de asumir el depósito de toda la producción de la universidad en que actúan. La manifestación del Investigador 3 corrobora lo que había sido afirmado por Joint (2006), que las bibliotecas y los bibliotecarios están allí para actuar, pero necesitan de inversiones para realizar un buen trabajo.

Además de inversiones en la biblioteca para que ellas puedan asumir el depósito de la producción científica en la institución, es necesaria inversión en mejorías en los repositorios, como es citado por los investigadores 5 y 8: el monitoreo del currículo Lattes, para que automáticamente los bibliotecarios sepan de las nuevas producciones que fueron publicadas, así como el mejoramiento de la interfaz de los repositorios, a fin de facilitar el proceso de depósito.

4.6. Las influencias positivas y negativas de los demás actores del sistema de comunicación científica

Los investigadores entrevistados fueron consultados sobre si podrían percibir una posible influencia, posi- tiva o negativa, de los demás actores que componen el sistema de comunicación científica (editores, agencias de fomento, bibliotecarios, gestores institucionales e investigadores), sobre su decisión en depositar o no la producción científica en el repositorio de su institución. En los resultados del análisis de los datos obtenidos por medio de las preguntas 7, 8, 9, 10 y 11 del guion de entrevistas de los investigadores están gráficamente representados en la Figura 3.

Cuando se preguntó sobre la influencia que los editores de revistas científicas ejercen sobre su decisión en depositar en los repositorios institucionales, todos los investigadores entrevistados, con la salvedad del Investigador 3, señalaron solamente influencias negativas, las cuales son causadas principalmente por los aspectos relacionados con la propiedad intelectual y con derechos de autor. Como el autor, en la mayoría de los casos en que va a publicar un artículo o un libro por una editora, cede los derechos de explotación de la obra, es el editor quien determina cuál uso se podrá hacer de aquella obra.

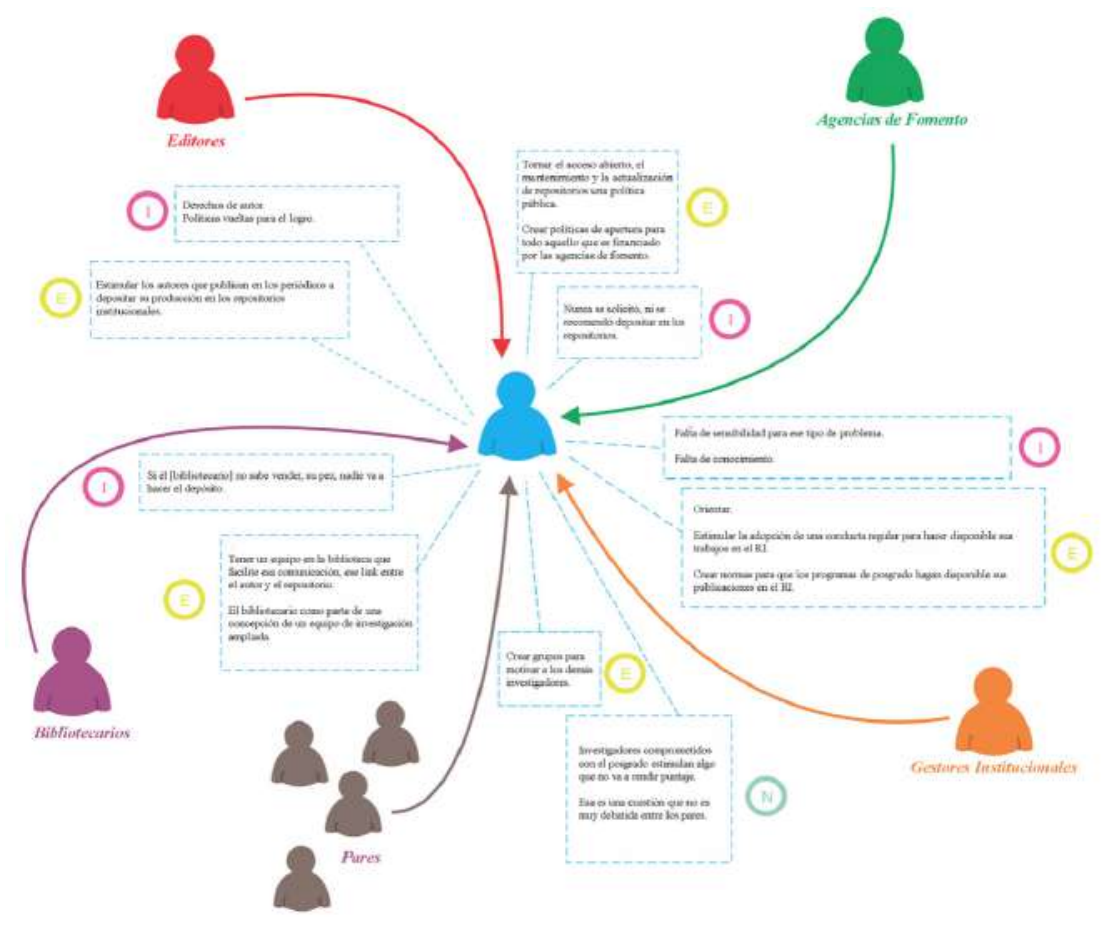

Figura 3. La percepción de los investigadores sobre la influencia de los distintos actores que participan del sistema comunicación científica.

Fuente: elaboración propia. 
En muchos casos en que el editor retiene el derecho de explotación de la obra, no permite que esté disponible en acceso abierto o, si lo permite, coloca una serie de restricciones como un periodo de embargo y la limitación de las versiones que pueden estar disponibles. Además de eso, los investigadores tienen dudas de lo que puede o no ser depositado en un repositorio institucional. Eso ocurre porque muchos, si no la mayoría de los contratos de derechos de autor, no lo mencionan o son ambiguos respecto a los derechos de autoarchivo y también sobre la cuestión de la versión que puede ser autoarchivada (Antelman, 2006).

Incluso las revistas científicas de acceso abierto, en muchos casos, no indican lo que se puede hacer con el contenido disponible en ellas. El hecho de que el contenido de la revista científica sea de acceso gratuito, no quiere decir que ese contenido pueda estar disponible en un repositorio institucional. Una forma de facilitar el de pósito de la producción científica publicada en esas revistas científicas es la adopción de licencias permisivas, como la Creative Commons, que expresan claramente lo que se puede hacer con el contenido publicado en la revista científica.

Los investigadores entrevistados vislumbran puntos positivos en la influencia que los gestores institucionales ejercen sobre su decisión de depositar en los repositorios institucionales. Esos gestores pueden influenciar positivamente en el momento en que se involucran con la divulgación del repositorio institucional, así como con la sensibilización del cuerpo docente sobre la importancia de hacer disponible su producción científica en acceso abierto.

El Manifiesto brasileño de apoyo al acceso libre a la información científica (IBICT, 2005) hace recomendaciones a las instituciones académicas para que también se comprometan a motivar a sus investigadores a publicar sus resultados de investigación en revistas científicas de acceso abierto, además de solicitar que depositen sus trabajos en el repositorio institucional de sus instituciones.

Sin embargo, los gestores institucionales que están al frente de la gestión de las universidades, en nivel de prorrectoría (decanato), dirección o coordinación de cursos de posgrado, son también investigadores y docentes de esa institución. En muchos casos, ni esos ges- tores poseen el conocimiento necesario para construir un trabajo de sensibilización. Así como es citado por la Investigadora 5 en su respuesta, esos gestores necesitan ser sensibilizados tanto como los demás investigadores de la universidad. Solamente a partir del momento que haya una comprensión por parte de los gestores institucionales acerca del papel de los repositorios institucionales y de los beneficios del acceso abierto para la comunidad académica, habrá un trabajo de divulgación y sensibilización por parte de esos gestores. Además de eso, serán creadas políticas de estímulo al depósito, creando mecanismos para que el investigador sea incentivado a depositar.

De esa forma, a partir del momento en que nada se hace por parte de las gestiones superiores de las universidades, el investigador sufre una influencia negativa, pues, si no existe divulgación, ni la motivación y tan poco hay algún tipo de política que estimule el depósito, difícilmente el investigador irá, sin la influencia de otros agentes, a tomar la decisión de depositar su producción en el repositorio de la institución. En ese punto, entra el papel fundamental de los bibliotecarios. El bibliotecario necesita dialogar con los gestores institucionales y eso es un gran desafío. De acuerdo con los investigadores entrevistados, prácticamente todos perciben la influencia positiva del bibliotecario en su decisión de depositar en el repositorio.

Se nota en las respuestas que aquellos investigadores que tienen parte de su producción científica depositada en el repositorio de su institución tuvieron un contacto previo con un bibliotecario. El papel del bibliotecario de divulgar y hacer marketing es crucial para el desarrollo de los repositorios institucionales. Antelman (2004) afirma que

los bibliotecarios que opten por implementar repositorios institucionales deben ser capaces de convencer a los profesores, muchos de los cuales son, por varias razones, muy resistentes a contribuir con su producción de investigación principal. Los datos muestran, sin embargo, que los artículos disponibles gratuitamente, por la mayor facilidad de uso, son más propensos a ser citados, lo que es una poderosa evidencia del valor de los repositorios, así como de otros canales de Acceso Abierto. (p. 374) 
Esta visión continúa válida actualmente. Los bibliotecarios aún necesitan ser capaces de convencer a los investigadores por medio de la divulgación del repositorio, presentando sus aspectos benéficos. Tal trabajo de divulgación es citado por prácticamente todos los investigadores como un factor que motiva su toma de decisiones para realizar el depósito.

Además de los factores motivacionales vinculados a la divulgación de los repositorios, principalmente atados al papel del gestor institucional y del bibliotecario, algunos investigadores afirman que las agencias de fomento podrían influenciar positivamente si adoptan políticas de incentivo a las publicaciones en acceso abierto. Actualmente, de acuerdo con algunos de los entrevistados, las agencias de fomento no estimulan nada, ni positiva ni negativamente, simplemente por el hecho de no tener ningún tipo de política enfocada en los repositorios institucionales.

Es importante resaltar que, en el Brasil, la Capes estipuló que las universidades públicas mantuvieran una versión en formato digital de las tesis y disertaciones defendidas a partir de 2006. Esa fue la primera iniciativa en términos de disponibilización de la información científica en acceso abierto en el Brasil.

El Investigador 7 afirmó que los repositorios institucionales deberían entrar como uno de los aspectos de impacto para evaluación del requisito "inserción social" de los cursos de posgrado stricto sensu. Ese requisito representa el $10 \%$ de la evaluación de cursos de maestría y doctorado académico y tiene como supuesto el hecho de que el posgrado tiene una responsabilidad social para con la sociedad. La inserción social tiende a evaluar cómo la investigación realizada en el ámbito de esos cursos actúa con relación con los desafíos decisivos para la sociedad. En concordancia con el Investigador 7, el repositorio institucional es un mecanismo de retorno de la academia para la sociedad, pues si aquellas investigaciones fueron financiadas con el dinero público, ellas deberían estar en acceso abierto para la comunidad local, el país y el mundo.

En ese sentido, las agencias de fomento, en la opinión de los investigadores, podrían crear mecanismos que no necesariamente obligaran al investigador a realizar el depósito, pero crearían los estímulos, en la evalua- ción de los cursos de posgrado o en las convocatorias de financiamiento de investigaciones, que servirían como factores motivacionales para la toma de decisiones al depositar su producción científica en repositorios institucionales.

Con relación a la influencia que puede ser ejercida por los pares, por otros investigadores del área, coautores, colaboradores, etc., solo dos afirmaron percibir alguna posible influencia. Los demás afirmaron que no perciben esa influencia, ni positiva ni negativa, pues ese asunto ni siquiera es debatido entre los investigadores.

Se entiende de este análisis que hay un desconocimiento por parte de los investigadores de lo que constituye un repositorio institucional. Tal desconocimiento se debe a la falta de divulgación y de adopción de políticas por parte de las universidades. Es recurrente en las declaraciones de todos los actores entrevistados que la cuestión del marketing y de la divulgación es crucial para el desarrollo de un repositorio, además de influenciar al investigador a optar por la diponibilidad de su producción científica en el repositorio de acceso abierto de su institución.

\section{Conclusiones}

El estudio realizado permitió concluir que el conocimiento de los investigadores entrevistados sobre acceso abierto converge con los principios céntricos del movimiento, cualesquiera que sean, la necesidad de remover las barreras del modelo tradicional de comunicación científica, así como deshacer las barreras que impiden el acceso a la literatura científica, a fin de acelerar la investigación, fortalecer la educación y difundir el conocimiento de manera general.

Las principales motivaciones para depositar la producción científica en repositorios institucionales son diversificadas en función de las áreas del conocimiento. Entre ellas están la conciencia de los beneficios del acceso abierto, la obligatoriedad del depósito, los estímulos institucionales y la vinculación a las disciplinas culturalmente propensas al depósito.

En lo que se refiere a los factores que estimulan o inhiben el depósito, se concluye que el principal estímulo, 
en la óptica de los investigadores, es la divulgación del repositorio y de los beneficios asociados al depósito de la producción científica en repositorios institucionales. Por otro lado, los principales factores que inhiben están relacionados con la falta de conocimiento sobre el repositorio institucional y con interrogaciones conectadas a la propiedad intelectual y los derechos de autor.

Independientemente del reconocimiento de la importancia de la obligatoriedad del depósito de la producción científica en repositorios institucionales, los resultados permiten concluir, a partir de la percepción de los investigadores entrevistados, que el camino más viable para hacer efectivo el depósito es la divulgación del servicio. En lo que concierne a la modalidad de depósito, se concluye que el depósito mediado corresponde a las principales expectativas de los investigadores.

Finalmente, los resultados permiten concluir que los investigadores se sienten influenciados positiva y negativamente por los demás factores estudiados. Sin embargo, con relación a sus pares, muchos investigadores afirmaron que no hay estímulos, ni positivos ni negativos, pues el tema "repositorios institucionales" ni siquiera es debatido entre ellos. En ese sentido, se percibe que hay desconocimiento por parte de los investigadores acerca del papel de los repositorios, y tal desconocimiento es atribuido a la falta de divulgación, así como a la falta de sensibilidad y orientación por parte de bibliotecarios y gestores institucionales. Las limitaciones relacionadas con las políticas editoriales que tienden al lucro, los derechos de autor y la falta de políticas públicas, por parte de las agencias de fomento, que tienden a la consolidación del acceso abierto, fueron citadas como factores que inhiben el depósito en repositorios institucionales. Es necesario crear estímulos para que el investigador deposite su producción en los repositorios institucionales. De acuerdo con los resultados, se concluye que cada actor, en correspondencia con sus funciones, puede estimular el depósito de la producción científica.

La decisión y la acción de depósito de la producción científica en repositorios institucionales por parte de investigadores depende, por lo tanto, de un conjunto de factores que están relacionados con todos los acto- res del sistema de comunicación científica. El conocimiento de tales factores es condición sine qua non para el establecimiento de estrategias que contribuyan al éxito del poblamiento de los repositorios institucionales de acceso abierto.

\section{Referencias}

1. Antelman, K. (2004). Do open-access articles have a greater research impact? College $\sim$ Research Libraries, 65(5), 372-382.

2. Antelman, K. (2006). Self-archiving practice and the influence of publisher policies in the social sciences. Learned Publishing, (19), 85-95. Recuperado de http://eprints.rclis. org/7420/1/antelman_self-archiving.pdf

3. Babbie, E. (1999). Métodos de Pesquisa de Survey. Belo Horizonte: UFMG.

4. BOAI. (2002). Budapest Open Access Initiative. Recuperado de http://www.budapestopenaccessinitiative.org/

5. Cooke, J. (2007). A visual arts perspective on open access institutional repositories. Proceedings of 23 Computers and History of Art Annual Conference. Birkbeck 8-9th November. Recuperado de http://research.gold.ac.uk/140/

6. Costa, S. M. S. (2005). A comunicação científica nos dias atuais: impactos de uma filosofia aberta. 57. ${ }^{\underline{a}}$ Reunião Anual da SBPC. Recuperado de http://www.sbpcnet.org. br/livro/57ra/programas/CONF_SIMP/textos/selycosta. htm

7. Covey, D. T. (2008). Faculty self-archiving practice: a case study. Selected works. Recuperado de https:// works.bepress.com/denise_troll_covey/35/ [Consulta: 23/04/2019].

8. Foster, N. F., \& Gibbons, S. (2005). Understanding faculty to improve content recruitment for institutional repositories. D-Lib Magazine, ll(1). Recuperado de http:// hdl.handle.net/1802/1292

9. Fowler, F. J. (2011). Pesquisa de levantamento. Porto Alegre: Penso.

10. Freitas, M. A. (2015). Diretrizes para o depósito da produção científica em repositorios institucionais (tesis de doctorado). Programa de Pós-Graduação em Ciência da Informação, Universidade de Brasília, Brasília.

11. Instituto Brasileiro de Informação em Ciência e Tecnologia (IBICT). (2005). Manifesto brasileiro de apoio ao acesso livre à informação cientifica. Recuperado de http:// livroaberto.ibict.br/Manifesto.pdf

12. Joint, N. (2006). Institutional repositories, selfarchiving and the role of the library. Library Review, 55. 
Recuperado de https://www.emeraldinsight.com/doi/ abs/10.1108/00242530610649576

13. Kankanhalli, A., Tan, B. C. Y., \& Wei, K.-K. (2005). Contributing knowledge to electronic knowledge repositories: an empirical investigation. CiteSeer. Recuperado de http://citeseerx.ist.psu.edu/viewdoc/ summary?doi=10.1.1.123.3555

14. Kim, J. (2008). Faculty self-archiving behavior: factors affecting the decision to self-archive (tesis de doctorado). Universidade de Michigan, Michigan. Recuperado de http://hdl.handle.net/2027.42/61564

15. Martins, A. B., Nolasco, B., \& Silva, D. (2013) Integração de informação e 176 interoperabilidade: o repositorio institucional como suporte à gestão da investigação na Universidade de Aveiro. R. Ci. Inf.e Doc., 4(2), 202-21l.

16. Xia, J., Gilchrist, S. B., Smith, N. X. P., Kingery, J. A., Radecki, J. R., Wilhelm, M. L.,... \& Mahn, A. J. (2012). A Review of Open Access Self-Archiving Mandate Policies. Libraries and the Academy, 12(1), 85-102. Recuperado de https://eric.ed.gov/?id=EJ975613

17. Xia, J., \& Sun, L. (2007). Factors to Assess Self-Archiving in Institutional Repositories. Serials Review, 33(2), 73-80. Recuperado de http://www.sciencedirect.com/science/ article/pii/S0098791306001365 